\title{
Generalized Klein-Gordon equation and quantum gravity corrections to tunneling of scalar particles from Kerr-Newman black hole
}

\author{
Aheibam Keshwarjit Singh ${ }^{1,2, a}{ }_{(\mathbb{D})}$, Irom Ablu Meitei ${ }^{3,4, b}$, Telem Ibungochouba Singh ${ }^{5, \mathrm{c}}$, \\ Kangujam Yugindro Singh ${ }^{1, d}$ \\ ${ }^{1}$ Department of Physics, Manipur University, Canchipur, Imphal, Manipur, India \\ 2 Department of Physics, Imphal College, Imphal, Manipur, India \\ ${ }^{3}$ Department of Physics, Dhanamanjuri College of Science, Imphal, Manipur, India \\ ${ }^{4}$ Visiting Associate in Inter-University Centre for Astronomy and Astrophysics, Pune, Maharashtra, India \\ 5 Department of Mathematics, Manipur University, Canchipur, Imphal, Manipur, India
}

Received: 4 March 2019 / Accepted: 30 July 2019 / Published online: 19 August 2019

(C) The Author(s) 2019

\begin{abstract}
In this article we deduce the generalized KleinGordon Equation in curved spacetime in the presence of an electromagnetic field from first principles, using the generalized uncertainty principle. Using this equation we study the tunneling of scalar particles from a Kerr-Newman black hole. Corrections to the Hawking temperature and entropy of the black hole due to quantum gravity effects are discussed.
\end{abstract}

\section{Introduction}

In early 1970 s the mathematical discovery of black hole area theorem gave a hint that there might be a relation between black hole and thermodynamics [1-3]. Bekenstein [4-6] provided an explanation for the interpretation of surface area of the event horizon as a measure of entropy of the black hole. In 1974, Hawking found that black holes emit particles at a steady rate and the outgoing particles have a precisely thermal spectrum $[7,8]$. This implied that a black hole could be in thermal equilibrium at some finite non-zero temperature. Hawking used the method of quantum field theory in curved spacetime to predict the existence of these radiations. Later different groups of researchers have developed other methods for obtaining the radiation.

\footnotetext{
a e-mail: a.keshwarjit@manipuruniv.ac.in

be-mail: ablu.irom@gmail.com

c e-mail: ibungochouba@ rediffmail.com

de-mail: yugindro361@gmail.com
}

Kraus et al. [9-13] developed a method whereby they considered Hawking radiation as a tunneling process. In their method the potential barrier is created by the outgoing particle and the radial null geodesic method in semiclassical WKB approximation is used. They also showed that a correction to the Hawking radiation spectrum appears when the backreaction effect of the tunneling particle is taken into account. Their method has been extended to other black holes [1419]. They have also shown that the spectrum is no longer precisely thermal.

Another tunneling approach is the Hamilton-Jacobi ansatz used by Agheben et al. [20], which is based on the complex path analysis developed by Padmanabhan et al. [21-24]. Agheben et al. investigated Hawking radiation as a tunneling of particles for the cases of rotating and extremal black hole using the relativistic Hamilton-Jacobi Equation and the WKB approximation. Since then other authors have applied the Hamilton-Jacobi method to study the Hawking radiation for other spacetimes [25-31].

To study Hawking radiation using tunneling methods one calculates the action for the emission process. Emission across the event horizon is a classically forbidden process and hence, the action is a complex quantity. Using the WKB approximation the tunneling probability for the emission process is

$\Gamma \sim \exp \left[-\frac{2}{\hbar} \operatorname{Im} I\right]$

where $I$ represents the classical action of the outgoing particle to leading order in $\hbar[32,33]$. 
In recent years there have been some progress in obtaining exact analytic solutions to Klein-Gordon equation in KerrNewman black hole background [34,35].

Various approaches to a quantum theory of gravity predict the existence of a minimal observable length [36-40]. This minimal length can be regarded as a minimal position uncertainty [41]. In this paper we study the effects of quantum gravity influenced by the Generalized Uncertainty Principle (GUP) and investigate the tunneling of scalar particles across the horizon of a (non-extremal) Kerr-Newman black hole by using the Hamilton-Jacobi ansatz. The black hole is considered to be in equilibrium with a scalar field at a temperature $T$ measured at infinity.

We find that the temperature of the black hole is affected by the mass, energy, angular momentum, charge and azimuthal angle of the emitted particle as well as the parameters of the black hole. For Kerr-Newman black hole, quantum gravity corrections slow down the rate of increase of the Hawking temperature.

Effects of GUP on tunneling of scalar particles for other black hole backgrounds are discussed in [42,43].

The paper is outlined as follows. In Sect. 2 we obtain the Generalized Klein Gordon Equation for a scalar particle in a curved spacetime background and interacting with an electromagnetic field. We briefly review the Kerr-Newman solution in the dragging coordinate system and the near horizon approximation in Sect. 3. In Sect. 4 we investigate Hawking radiation as tunneling of scalar particles across the event horizon of a Kerr-Newman black hole. In Sect. 5 we discuss the back-reaction of tunneling particles and the entropy correction. In Sect. 6 we present our discussion and conclusions.

In this paper we use the metric signature $(-,+,+,+)$. Latin index $k$ runs over values $0,1,2,3$. Greek indices $\mu, v$ run over $1,2,3$.

\section{Generalized Klein Gordon equation}

Theories of quantum gravity and string theory predict a nonzero minimal uncertainty $\Delta x_{0}$ in position measurements [3640]. Generalized uncertainty principle (GUP) is an effective way to realize this minimal length [41]. In one dimension the simplest generalized uncertainty relation has the form [44]:

$\Delta x \Delta p \geq \frac{\hbar}{2}\left[1+\beta(\Delta p)^{2}\right]$

where $\beta=\beta_{0} \ell_{P}^{2} / \hbar^{2} ; \beta_{0}$ is a dimensionless parameter of order unity when one considers Planck length, $\ell_{P}=$ $\sqrt{G \hbar / c^{3}}$, as the minimal length scale. Garay [39], in a series of arguments, showed that $\Delta x \geq \max \left(\frac{1}{\Delta p}, \ell^{2}{ }_{P} \Delta p\right)$, where $\hbar=c=1$. Following a simple and general consideration of the Newtonian gravitational theory, Adler and Santiago
[45] obtained a modified uncertainty relation in agreement with the approximate order of $\beta_{0}$ (i.e. of order one). A similar result is also obtained using linearized general relativity. By computing the Hawking temperature of a Schwarzschild black hole in two different ways - first, by using the GUP to compute the Hawking temperature containing correction term depending on $\beta_{0}$ and second, by considering quantum correction to the Newtonian potential - and then comparing the two results, Scardigli et al. [46] obtained a precise numerical value of $\beta_{0}$, viz. $\beta_{0}=82 \pi / 5$, which is of order one. Because of the GUP one can define the generalized frequency, $\tilde{\omega}$, as $[47,48]$

$\hbar \tilde{\omega}=E\left(1-\beta E^{2}\right)$.

Using the energy mass shell condition, $E^{2}=p^{2}+m^{2}$, we get

$\tilde{E}^{2}=\left(p^{2}+m^{2}\right)\left[1-2 \beta\left(p^{2}+m^{2}\right)\right]$

up to first order in $\beta$ and second order in $p$. For a particle of charge $q$ influenced by a vector field $A_{k}=(-\Phi, \mathbf{A})$, we use the minimal substitution $[49,50]$

$p_{k} \rightarrow p_{k}-q A_{k}, \quad p^{k} \rightarrow p^{k}-q A^{k}$

in Eq. 4. Using the operator representation for 4-momentum, we obtain the generalized Klein-Gordon Equation in the presence of an electromagnetic field:

$$
\begin{aligned}
& -\left(-i \hbar \partial^{t}-q A^{0}\right)\left(-i \hbar \partial_{t}-q A_{0}\right) \Psi \\
& =\left[\left(-i \hbar \partial^{\mu}-q A^{\mu}\right)\left(-i \hbar \partial_{\mu}-q A_{\mu}\right)+m^{2}\right] \\
& \quad \times\left[1-2 \beta\left\{\left(-i \hbar \partial^{v}-q A^{v}\right)\left(-i \hbar \partial_{v}-q A_{\nu}\right)+m^{2}\right\}\right] \Psi
\end{aligned}
$$

where $\mu, v=1,2,3$.

\section{Kerr-Newman black hole}

The metric of Kerr-Newman black hole in Boyer-Lindquist coordinates is given by $(G=1=c)$ [51]

$$
\begin{aligned}
d s^{2}= & -\left(\frac{\Delta-a^{2} \sin ^{2} \theta}{\Sigma}\right) d t^{2} \\
& -\frac{2 a \sin ^{2} \theta\left(r^{2}+a^{2}-\Delta\right)}{\Sigma} d t d \phi \\
& +\frac{\Sigma}{\Delta} d r^{2}+\Sigma d \theta^{2} \\
& +\left[\frac{\left(r^{2}+a^{2}\right)^{2}-\Delta a^{2} \sin ^{2} \theta}{\Sigma}\right] \sin ^{2} \theta d \phi^{2},
\end{aligned}
$$

where $\Sigma=r^{2}+a^{2} \cos ^{2} \theta$, and $\Delta=r^{2}+a^{2}-2 M r+Q^{2}$. 
$M, a, Q$ are the parameters of the black hole representing mass, angular momentum per unit mass and electric charge respectively. An appropriate four potential of the electromagnetic field is $[51,52]$

$A_{0}=\frac{-Q r}{\Sigma}, \quad A_{1}=0, \quad A_{2}=0, \quad A_{3}=\frac{Q r a \sin ^{2} \theta}{\Sigma}$.

Because of rotation, the event horizon does not coincide with the infinite red-shift surface in this coordinate system. Hence, the geometrical optical limit cannot be used here and the WKB approximation is not valid. This hindrance can be removed by performing a transformation to a frame which is rotating with the black hole.

\subsection{Frame dragging}

A rotating black hole drags inertial frames around it. This forces particles and photons in the ergosphere to orbit the black hole with an angular velocity $\Omega \equiv \frac{\mathrm{d} \phi}{\mathrm{d} t}>0$. A locally non rotating observer is one who moves with coordinate angular velocity $\Omega=-\frac{g_{03}}{g_{33}}[51,53,54]$. In the frame of such an observer the line element is [55]

$d s^{2}=-\frac{\Sigma \Delta}{\left(r^{2}+a^{2}\right)^{2}-\Delta a^{2} \sin ^{2} \theta} d t^{2}+\frac{\Sigma}{\Delta} d r^{2}+\Sigma d \theta^{2}$.

The corresponding vector potential has only one non vanishing component $A_{0}$ given by

$A_{0}=\frac{-\left(r^{2}+a^{2}\right) Q r}{\left(r^{2}+a^{2}\right)^{2}-\Delta a^{2} \sin ^{2} \theta}$.

\subsection{Near horizon approximation}

Assuming that the black hole is non-extremal so that $M^{2}>$ $Q^{2}-a^{2}$, the horizons, defined by the equation $\Delta=0$, are located at

$r_{ \pm}=M \pm \sqrt{M^{2}-Q^{2}-a^{2}}$

where $r_{+}$and $r_{-}$are called the outer horizon and the inner horizon respectively. The outer horizon is the one that is relevant from the point of view of an external observer [49]. Henceforth we will refer to the outer horizon as the event horizon. The dragging coordinate transformation makes the event horizon coincide with the infinite redshift surface $\left(g_{00}=0\right)$ so that the geometrical optical limit becomes a reliable approximation and the WKB approximation can be applied.
Near the event horizon $r=r_{+}$we use the approximation

$$
\begin{aligned}
\Delta(r) & =\Delta\left(r_{+}\right)+\left(r-r_{+}\right) \Delta,{ }_{r}\left(r_{+}\right)+\mathcal{O}\left(\left(r-r_{+}\right)^{2}\right) \\
& \approx\left(r-r_{+}\right) \Delta,{ }_{r}\left(r_{+}\right) .
\end{aligned}
$$

The line element Eq. 9 takes the form

$$
\begin{aligned}
d s^{2}= & -\frac{\Sigma\left(r_{+}\right) \Delta, r\left(r_{+}\right)\left(r-r_{+}\right)}{\left(r_{+}^{2}+a^{2}\right)^{2}} d t^{2} \\
& +\frac{\Sigma\left(r_{+}\right)}{\Delta, r\left(r_{+}\right)\left(r-r_{+}\right)} d r^{2}+\Sigma\left(r_{+}\right) d \theta^{2} .
\end{aligned}
$$

The non-vanishing component of vector potential at the event horizon is

$A_{0}=\frac{-Q r_{+}}{r_{+}^{2}+a^{2}}$.

\section{Quantum tunneling from Kerr-Newman black hole}

We consider the tunneling of scalar particles from KerrNewman black hole. The motion of scalar particles obey the generalized Klein-Gordon Equation given in Eq. 6. Using the inverse metric obtained from Eq. 11 into Eq. 6, we get

$$
\begin{aligned}
& \frac{\left(r_{+}^{2}+a^{2}\right)^{2}}{\Sigma\left(r_{+}\right) \Delta, r\left(r_{+}\right)\left(r-r_{+}\right)}\left(-i \hbar \partial_{t}-q A_{0}\right)^{2} \Psi \\
& =\left[\frac{\Delta, r\left(r_{+}\right)\left(r-r_{+}\right)}{\Sigma\left(r_{+}\right)}\left(-i \hbar \partial_{r}\right)^{2}+\frac{1}{\Sigma\left(r_{+}\right)}\left(-i \hbar \partial_{\theta}\right)^{2}+m^{2}\right] \\
& \quad \times\left[1-2 \beta\left\{\frac{\Delta, r\left(r_{+}\right)\left(r-r_{+}\right)}{\Sigma\left(r_{+}\right)}\left(-i \hbar \partial_{r}\right)^{2}\right.\right. \\
& \left.\left.\quad+\frac{1}{\Sigma\left(r_{+}\right)}\left(-i \hbar \partial_{\theta}\right)^{2}+m^{2}\right\}\right] \Psi .
\end{aligned}
$$

The semiclassical wavefunctions satisfying the above equation are obtained by making the standard ansatz for $\Psi$ :

$\Psi=\exp \left[\frac{i}{\hbar} S(t, r, \theta)\right]$

Using the WKB approximation in Eq. 12 we obtain the Generalized Hamilton-Jacobi Equation

$$
\begin{aligned}
-g^{00}\left(\frac{\partial I}{\partial t}-q A_{0}\right)^{2} \\
=\left[g^{11}\left(\frac{\partial I}{\partial r}\right)^{2}+g^{22}\left(\frac{\partial I}{\partial \theta}\right)^{2}+m^{2}\right] \\
\quad \times\left[1-2 \beta\left\{g^{11}\left(\frac{\partial I}{\partial r}\right)^{2}+g^{22}\left(\frac{\partial I}{\partial \theta}\right)^{2}+m^{2}\right\}\right],
\end{aligned}
$$

where $I(t, r, \theta)$ is classical action of the scalar particle to leading order of $\hbar$. Henceforth, we use Planck units $(G=$ $c=\hbar=1$ ) for simplicity. 
Considering the symmetry of the black hole, the action of the scalar particle may be taken in the form

$$
I=-(\omega-j \Omega) t+R(r)+W(\theta)+K
$$

where $\omega$ is the energy of the particle, $j$ the angular momentum with respect to the angle $\phi$ and $K$ a constant which may be complex. Substituting Eq. 15 into the Generalized HamiltonJacobi Equation 14 and solving for $R(r)$ we obtain

$$
\begin{aligned}
R(r)_{ \pm}= & \pm \int \frac{r_{+}^{2}+a^{2}}{2\left(r-r_{+}\right)\left(r_{+}-M\right)} \sqrt{C-\left(V+m^{2}\right) F} \\
& \times\left[1+\frac{\beta C^{2}}{F^{2}\left(C / F-V-m^{2}\right)}\right] d r
\end{aligned}
$$

where

$$
\begin{aligned}
& C=\left(-\omega+j \Omega_{h}-q A_{0}\right)^{2}, \\
& F=-\frac{1}{g^{00}}=\frac{\Sigma\left(r_{+}\right) \Delta, r\left(r_{+}\right)\left(r-r_{+}\right)}{\left(r_{+}^{2}+a^{2}\right)^{2}}, \\
& V=g^{22}\left(\frac{\mathrm{d} W}{\mathrm{~d} \theta}\right)^{2}=\frac{1}{\Sigma\left(r_{+}\right)}\left(\frac{\mathrm{d} W}{\mathrm{~d} \theta}\right)^{2} .
\end{aligned}
$$

The sign ambiguity is related to the outgoing (particle moving away from the black hole) or ingoing (particle approaching the black hole) nature of the particle. The \pm sign corresponds to outgoing and incoming wave respectively. The residue at the pole $r=r_{+}$is calculated for the outgoing/incoming particle and we obtain

$$
\begin{aligned}
R_{ \pm}= & \pm \frac{i \pi\left(r_{+}^{2}+a^{2}\right)}{2\left(r_{+}-M\right)}\left(\omega-j \Omega_{h}+q A_{0}\right) \\
& \times\left[1+\left(m^{2}+\frac{\left(\frac{\mathrm{d} W}{\mathrm{~d} \theta}\right)^{2}}{r_{+}^{2}+a^{2} \cos ^{2}(\theta)}\right) \frac{\beta}{2}\right] .
\end{aligned}
$$

Therefore, from Eq. 15

$$
\begin{aligned}
I & =-(\omega-j \Omega) t+R_{ \pm}+W(\theta)+K \\
& =R_{ \pm}+K+\text { (real parts). }
\end{aligned}
$$

\subsection{Emission rate of the black hole}

The emission rate is the tunneling probability for an outgoing positive energy particle. Using the WKB approximation the tunneling probabilities are:

$$
\begin{aligned}
\mathrm{P}[\text { emission }] & \sim \exp \left[-2\left(\operatorname{Im} R_{+}+\operatorname{Im} K\right)\right] \\
\mathrm{P}[\text { absorption }] & \sim \exp \left[-2\left(\operatorname{Im} R_{-}+\operatorname{Im} K\right)\right] .
\end{aligned}
$$

The tunneling rate [56] of the scalar particle crossing from inside to outside the black hole event horizon is

$$
\begin{aligned}
\Gamma= & \exp \left[-\frac{2 \pi\left(r_{+}^{2}+a^{2}\right)}{\left(r_{+}-M\right)}\left(\omega-j \Omega_{h}+q A_{0}\right)\right. \\
& \left.\times\left\{1+\frac{\beta}{2}\left(m^{2}+\frac{\left(\frac{\mathrm{d} W}{\mathrm{~d} \theta}\right)^{2}}{r_{+}^{2}+a^{2} \cos ^{2}(\theta)}\right)\right\}\right] .
\end{aligned}
$$

The Boltzmann factor yields the Hawking temperature $[20,55,57]$. The Hawking temperature of the Kerr-Newman black hole is obtained as:

$$
\begin{aligned}
T & =\frac{\left(r_{+}-M\right)}{2 \pi\left(r_{+}^{2}+a^{2}\right)}\left[1+\left(m^{2}+\frac{\left(\frac{\mathrm{d} W}{\mathrm{~d} \theta}\right)^{2}}{r_{+}^{2}+a^{2} \cos ^{2} \theta}\right) \frac{\beta}{2}\right]^{-1} \\
& =T_{0}\left[1-\left(m^{2}+\frac{\left(\frac{\mathrm{d} W}{\mathrm{~d} \theta}\right)^{2}}{r_{+}^{2}+a^{2} \cos ^{2} \theta}\right) \frac{\beta}{2}\right]
\end{aligned}
$$

where $T_{0}=\frac{\left(r_{+}-M\right)}{2 \pi\left(r_{+}^{2}+a^{2}\right)}=\frac{\left(r_{+}-r_{-}\right)}{4 \pi\left(r_{+}^{2}+a^{2}\right)}$ is the original Hawking temperature of a Kerr-Newman black hole without any quantum gravity correction. The corrected temperature is lower than the original temperature $T_{0}$. The corrected temperature depends on the mass of the emitted scalar particle, the azimuthal angle $\theta$ and $\frac{d W}{d \theta}$. The result we have obtained is in agreement with the one obtained by Wang [43]. Because of the quantum gravity effects the rate of increase of temperature during radiation is slowed down. In the absence of quantum gravity effects, i.e., $\beta=0$, the corrected Hawking temperature reduces to the original Hawking temperature as is evident from Eq. 23.

The explanation for the dependence on $\theta$ is as follows: Consider the Kerr-Newman black hole as a charged sphere rotating about a fixed axis. Because of rotating charges it produces a magnetic field which is axisymmetric, i.e., it depends on $\theta$ but not on $\phi$. As a result a charged particle experiences a Lorentz force which is dependent on $\theta$. Further, Lorentz force is dependent on the particle's velocity which explains the dependence on $\frac{d W}{d \theta}$.

\subsection{Second approximation}

Here we take the near horizon approximation as

$F=\frac{\Sigma\left(r_{+}\right) \Delta(r)}{\left(r_{+}^{2}+a^{2}\right)^{2}} ; \quad G=g^{11}=\frac{\Delta(r)}{\Sigma\left(r_{+}\right)}$.

Under the condition $\left|\frac{\left(-\omega+j \Omega-q A_{0}\right)^{2}}{m^{2}+V}\right| \gg|F|$ the integral Eq. 16 is approximated as

$$
\begin{aligned}
R(r)_{ \pm}= & \pm \int \frac{1}{\sqrt{F G}} \sqrt{C-\left(V+m^{2}\right) F} \\
& \times\left[1+\beta\left(m^{2}+V\right)+\frac{\beta C}{F}\right] d r
\end{aligned}
$$


which yields

$R_{ \pm}= \pm \frac{i \pi\left(r_{+}^{2}+a^{2}\right)}{2\left(r_{+}-M\right)}\left(\omega-j \Omega_{h}+q A_{0}\right)[1+\beta \Pi]$,

where $\Pi=\frac{1}{2}\left(m^{2}+\frac{\left(\frac{\mathrm{d} W}{\mathrm{~d} \theta}\right)^{2}}{r_{+}^{2}+a^{2} \cos ^{2} \theta}-\frac{\left(\omega-j \Omega_{h}+q A_{0}\right)^{2}\left(r_{+}^{2}+a^{2}\right)^{2}}{3\left(r_{+}^{2}+a^{2} \cos ^{2} \theta\right)\left(r_{+}-M\right)^{2}}\right)$. The tunneling rate and the temperature of the black hole horizon are respectively

$\Gamma=\exp \left[-\frac{2 \pi\left(r_{+}^{2}+a^{2}\right)}{\left(r_{+}-M\right)}\left(\omega-j \Omega_{h}+q A_{0}\right)(1+\beta \Pi)\right]$

and

$T=\frac{T_{0}}{1+\beta \Pi}$.

In this case the corrected Hawking temperature depends not only on the mass of the black hole but also on the mass, energy, angular momentum, charge and azimuthal angle of the emitted particle. Thus, the Hawking temperature and hence, the thermodynamics of black hole is related indirectly to the curvature of spacetime, which is in agreement with [58].

\section{Self-gravitation interaction and entropy correction}

So far we have neglected the effect of back-reaction of the tunneling particle. To take this into account we consider a scalar particle tunneling out of the black hole horizon. As the total energy, total angular momentum and total electric charge of the black hole and emitted particle must be conserved, the outgoing particle must tunnel out a radial barrier to a locally non rotating observer [59]. The black hole will shrink due to the loss of energy, angular momentum, and electric charge. Its angular velocity as well as its electric potential at the horizon will change. This means the geometry is dynamic. This picture enables us to compute the change in entropy, tunneling rate and the radiant spectrum of a Kerr-Newman black hole for the case of scalar particle emission. We will show that when self-gravitation interaction is taken into consideration, the tunneling probability, in the presence of quantum gravity effects, is related to the change of Bekenstein-Hawking entropy.

When a black hole emits a particle with energy $\omega$, charge $q$ and angular momentum $j$, the black hole parameters change to $M-\omega, Q-q, J-j$ respectively [55]. The imaginary part of the action given in Eq. 19 then becomes:

$$
\begin{aligned}
\operatorname{Im} I= & \int_{(0,0,0)}^{(\omega, q, j)} \frac{\pi\left(r_{+}^{\prime 2}+a^{\prime 2}\right)}{r_{+}^{\prime}-\left(M-\omega^{\prime}\right)}\left(d \omega^{\prime}-\Omega_{h}^{\prime} d j^{\prime}-d q^{\prime} \Phi_{h}^{\prime}\right) \\
& \times\left[1+\left(m^{2}+\frac{\left(\frac{\mathrm{d} W}{\mathrm{~d} \theta}\right)^{2}}{r_{+}^{\prime 2}+a^{\prime 2} \cos ^{2}(\theta)}\right) \frac{\beta}{2}\right] \\
= & -\int_{(M-\omega, Q-q, J-j)}^{\pi\left(r_{+}^{\prime 2}+a^{\prime 2}\right)} \frac{\int_{+}^{\prime}-\left(M-\omega^{\prime}\right)}{\left.r_{+}^{\prime}-J\right)} \\
& \times\left[d\left(M-\omega^{\prime}\right)-\Omega_{h}^{\prime} d\left(J-j^{\prime}\right)\right. \\
& \left.\times-\frac{\left(Q-q^{\prime}\right) r_{+}^{\prime} d\left(Q-q^{\prime}\right)}{r_{+}^{\prime 2}+a^{\prime 2}}\right] \\
& \times\left[1+\frac{\beta}{2}\left(m^{2}+\frac{\left(\frac{\mathrm{d} W}{\mathrm{~d} \theta}\right)^{2}}{r_{+}^{\prime 2}+a^{\prime 2} \cos ^{2}(\theta)}\right)\right]
\end{aligned}
$$

where

$\Omega_{h}^{\prime}=\frac{a^{\prime}}{r_{+}^{\prime 2}+a^{\prime 2}}, \quad J-j^{\prime}=\left(M-\omega^{\prime}\right) a^{\prime}, \quad \Phi_{h}^{\prime}=\frac{\left(Q-q^{\prime}\right) r_{+}^{\prime}}{r_{+}^{\prime 2}+a^{\prime 2}}$,

$r_{+}^{\prime}=\left(M-\omega^{\prime}\right)+\sqrt{\left(M-\omega^{\prime}\right)^{2}-\left(Q-q^{\prime}\right)^{2}-a^{\prime 2}}$.

Thus the imaginary part of the action is

$$
\begin{aligned}
\operatorname{Im} I= & -\frac{\pi}{2}\left[\left(r_{f}^{2}+a_{f}^{2}\right)-\left(r_{i}^{2}+a_{i}^{2}\right)\right] \\
& -\frac{\pi \beta}{4} \int_{\left(r_{i}, a_{i}\right)}^{\left(r_{f}, a_{f}\right)} d\left(r_{+}^{\prime 2}+a^{\prime 2}\right)\left(m^{2}+\frac{\left(\frac{\mathrm{d} W}{\mathrm{~d} \theta}\right)^{2}}{r_{+}^{\prime 2}+a^{\prime 2} \cos ^{2} \theta}\right) .
\end{aligned}
$$

Considering emission along the axis of rotation, i.e. $\theta=0, \pi$, we get

$$
\begin{aligned}
\operatorname{Im} I= & -\frac{\pi}{2}\left[\left(r_{f}^{2}+a_{f}^{2}\right)-\left(r_{i}^{2}+a_{i}^{2}\right)\right] \\
& -\frac{m^{2} \pi \beta}{4}\left[\left(r_{f}^{2}+a_{f}^{2}\right)-\left(r_{i}^{2}+a_{i}^{2}\right)\right] \\
& -\frac{\pi \beta}{4}\left(\frac{\mathrm{d} W}{\mathrm{~d} \theta}\right)^{2}\left[\ln \left(\pi\left(r_{f}^{2}+a_{f}^{2}\right)\right)-\ln \left(\pi\left(r_{i}^{2}+a_{i}^{2}\right)\right)\right] .
\end{aligned}
$$

\subsection{Entropy correction}

To calculate the change in entropy let us consider a quasistatic process in which a Kerr-Newman black hole in an initial equilibrium state at temperature $T$ emits a scalar particle of infinitesimal mass, angular momentum and charge, and settles to a nearby final equilibrium state. The first law of black hole thermodynamics then relates the change in the black hole mass $\mathrm{M}$ to the changes in its entropy $S_{b h}$, angular momentum $J$ and electric charge $Q$ as $[5,60]$

$T d S_{b h}=d M-\Omega_{h} d J-\Phi_{h} d Q$. 
The angular frequency $\Omega_{h}$ and the scalar potential $\Phi_{h}$ are constant over the event horizon of the black hole. Then the entropy change during a finite change from an initial state "i" to a final state "f" is

$$
\begin{aligned}
\Delta S_{b h}= & \int_{i}^{f} \frac{1}{T}\left(d M-\Omega_{h} d J-\Phi_{h} d Q\right) \\
= & \Delta S_{B H} \\
& +\frac{\pi \beta}{2} \int_{\left(r_{i}, a_{i}\right)}^{\left(r_{f}, a_{f}\right)} d\left(r_{+}^{2}+a^{2}\right)\left(m^{2}+\frac{\left(\frac{\mathrm{d} W}{\mathrm{~d} \theta}\right)^{2}}{r_{+}^{2}+a^{2} \cos ^{2} \theta}\right),
\end{aligned}
$$

where $\Delta S_{B H}=S_{B H}(M-\omega, J-j, Q-q)-S_{B H}(M, J, Q)$ $=\pi\left[\left(r_{f}^{2}+a_{f}^{2}\right)-\left(r_{i}^{2}+a_{i}^{2}\right)\right]$ is the change in BekensteinHawking entropy of the Kerr-Newman black hole. As done earlier we consider emission along the axis of rotation $\theta=$ $0, \pi$ and obtain the expression

$$
\Delta S_{b h}=\Delta S_{B H}+\frac{m^{2} \beta}{2} \Delta S_{B H}+\frac{\pi \beta}{2}\left(\frac{\mathrm{d} W}{\mathrm{~d} \theta}\right)^{2} \Delta \ln S_{B H}
$$

where $\Delta \ln S_{B H}=\left[\ln \left(\pi\left(r_{f}^{2}+a_{f}^{2}\right)\right)-\ln \left(\pi\left(r_{i}^{2}+a_{i}^{2}\right)\right)\right]$. Thus, quantum gravity effect introduces correction terms proportional to $\Delta S_{B H}$ and $\Delta \ln S_{B H}$ respectively. Correction terms proportional to $\Delta S_{B H}$ and $\Delta \ln S_{B H}$ have been obtained in earlier literatures too [61-63].

Further, from Eqs. 30 and 32 we see that

$\Delta S_{b h}=-2 \operatorname{Im} I$.

Hence, in the WKB approximation, the tunneling rate of the scalar particle is

$\Gamma=\exp [-2 \operatorname{Im} I]=\exp \left[\Delta S_{b h}\right]$.

Thus, the tunneling rate of scalar particles through the event horizon of the Kerr-Newman black hole is related to the change in entropy of the black hole when self gravitation is taken into account. Quantum gravity corrections introduce extra terms $\exp \left(\frac{m^{2} \beta}{2} \Delta S_{B H}\right)$ and $\exp \left(\frac{\pi \beta}{2}\left(\frac{\mathrm{d} W}{\mathrm{~d} \theta}\right)^{2} \Delta \ln S_{B H}\right)$ which slow down the rate of particle emission during the tunneling process.

\section{Discussion and conclusion}

In this work we have obtained the generalized Klein-Gordon Equation in curved space-time in the presence of an electromagnetic field by employing the generalized uncertainty principle. We use this generalized equation to study the effects of quantum gravity to the tunneling of charged scalar particles from Kerr-Newman black hole. The tunneling rate of particles across the horizon and consequently the temperature of the black hole horizon is then calculated using the WKB approximation. It is found that the corrected Hawking temperature depends on the parameters of the black hole as well as the energy, mass, spin, charge and azimuthal angle of the emitted particle. Thus, the Hawking temperature and hence, the thermodynamics of black hole is related to the quantum numbers of the emitted particle and indirectly to the curvature of spacetime. Our work shows that the temperature is reduced due to quantum gravity correction which slows the tunneling rate.

By taking the particle's self gravitation into account along with the conservation of energy, angular momentum and charge we have calculated the transmission probability. Further, the entropy change of the Kerr-Newman black hole during the emission of scalar particle is also calculated. We have found that the tunneling rate of scalar particle is related to change in entropy of the black hole during the emission of the particle, and the radiation spectrum is not purely thermal. Quantum gravity influence introduces correction terms $\frac{m^{2} \beta}{2} \Delta S_{B H}$ and $\frac{\pi \beta}{2}\left(\frac{\mathrm{d} W}{\mathrm{~d} \theta}\right)^{2} \Delta \ln S_{B H}$ to the BekensteinHawking entropy. This correction terms increase the magnitude of change in entropy of the black hole and the tunneling rate is slowed down.

Data Availability Statement This manuscript has no associated data or the data will not be deposited. [Authors' comment: The paper is a theoretical work and no experimental or observational data is associated with it.]

Open Access This article is distributed under the terms of the Creative Commons Attribution 4.0 International License (http://creativecomm ons.org/licenses/by/4.0/), which permits unrestricted use, distribution, and reproduction in any medium, provided you give appropriate credit to the original author(s) and the source, provide a link to the Creative Commons license, and indicate if changes were made. Funded by SCOAP $^{3}$.

\section{References}

1. S.W. Hawking, Phys. Rev. Lett. 26, 1344 (1971). https://doi.org/ 10.1103/PhysRevLett.26.1344

2. S.W. Hawking, Commun. Math. Phys. 25, 152 (1972). https://doi. org/10.1007/BF01877517

3. S.W. Hawking, Sci. Am. 236, 34 (1977). https://doi.org/10.1038/ scientificamerican0177-34

4. J.D. Bekenstein, Lett. Nuovo Cim. 4, 737 (1972). https://doi.org/ 10.1007/BF02757029

5. J.D. Bekenstein, Phys. Rev. D 7, 2333 (1973). https://doi.org/10. 1103/PhysRevD.7.2333

6. J.D. Bekenstein, Phys. Rev. D 9, 3292 (1974). https://doi.org/10. 1103/PhysRevD.9.3292

7. S.W. Hawking, Nature 248, 30 (1974). https://doi.org/10.1038/ 248030a0

8. S.W. Hawking, Commun. Math. Phys. 43, 199 (1975). https://doi. org/10.1007/BF02345020, https://doi.org/10.1007/BF01608497 
9. M.K. Parikh, F. Wilczek, Phys. Rev. Lett. 85, 5042 (2000). https:// doi.org/10.1103/PhysRevLett.85.5042

10. P. Kraus, F. Wilczek (1994) arXiv:gr-qc/9406042

11. P. Kraus, F. Wilczek, Nucl. Phys. B 433, 403 (1995). https://doi. org/10.1016/0550-3213(94)00411-7

12. P. Kraus, F. Wilczek, Nucl. Phys. B 437, 231 (1995). https://doi. org/10.1016/0550-3213(94)00588-6

13. E. Keski-Vakkuri, P. Kraus, Nucl. Phys. B 491, 249 (1997). https:// doi.org/10.1016/S0550-3213(97)00085-0

14. E.C. Vagenas, Phys. Lett. B 559, 65 (2003). https://doi.org/10. 1016/S0370-2693(03)00302-2

15. J.Y. Zhang, Z. Zhao, Mod. Phys. Lett. A 20, 1673 (2005). https:// doi.org/10.1142/S0217732305017019

16. J.Y.Zhang, Z. Zhao, JHEP 10, 055 (2005). https://doi.org/10.1088/ $1126-6708 / 2005 / 10 / 055$

17. J.Y. Zhang, Z. Zhao, Phys. Lett. B 618, 14 (2005). https://doi.org/ 10.1016/j.physletb.2005.05.024

18. J. Zhang, Z. Zhao, Phys. Lett. B 638, 110 (2006). https://doi.org/ 10.1016/j.physletb.2006.05.059

19. R. Banerjee, B.R. Majhi, Phys. Lett. B 662, 62 (2008). https://doi. org/10.1016/j.physletb.2008.02.044

20. M. Angheben, M. Nadalini, L. Vanzo, S. Zerbini, JHEP 05, 014 (2005). https://doi.org/10.1088/1126-6708/2005/05/014

21. K. Srinivasan, T. Padmanabhan, Phys. Rev. D 60, 024007 (1999). https://doi.org/10.1103/PhysRevD.60.024007

22. S. Shankaranarayanan, K. Srinivasan, T. Padmanabhan, Mod. Phys. Lett. A 16, 571 (2001). https://doi.org/10.1142/ S0217732301003632

23. S. Shankaranarayanan, T. Padmanabhan, K. Srinivasan, Class. Quantum Gravity 19, 2671 (2002). https://doi.org/10.1088/ 0264-9381/19/10/310

24. S. Shankaranarayanan, Phys. Rev. D 67, 084026 (2003). https:// doi.org/10.1103/PhysRevD.67.084026

25. J. Ren, Z. Zhao, Int. J. Theor. Phys. 45, 1181 (2006). https://doi. org/10.1007/s10773-006-9101-8

26. J. Ren, Z. Zhao, Int. J. Theor. Phys. 46, 3109 (2007). https://doi. org/10.1007/s10773-007-9424-0

27. G. Wang, B. Liu, W. Liu, Gen. Rel. Gravit. 42, 633 (2010). https:// doi.org/10.1007/s10714-009-0876-9

28. Y.P. Hu, J.Y. Zhang, Z. Zhao, Mod. Phys. Lett. A 25, 295 (2010). https://doi.org/10.1142/S0217732310031713

29. M.A. Rahman, M.I. Hossain, Phys. Lett. B 712, 1 (2012). https:// doi.org/10.1016/j.physletb.2012.04.049

30. T.I. Singh, I.A. Meitei, K.Y. Singh, Astrophys. Space Sci. 352(2), 737 (2014). https://doi.org/10.1007/s10509-014-1927-6

31. T.I. Singh, I.A. Meitei, K.Y. Singh, Astrophys. Space Sci. 361(3), 103 (2016). https://doi.org/10.1007/s10509-016-2691-6

32. R. Kerner, R.B. Mann, Phys. Rev. D 73(10), 104010 (2006). https:// doi.org/10.1103/PhysRevD.73.104010

33. R. Kerner, R.B. Mann, Class. Quantum Gravity 25(9), 095014 (2008). https://doi.org/10.1088/0264-9381/25/9/095014

34. V.B. Bezerra, H.S. Vieira, A.A. Costa, Class. Quant. Grav. 31(4), 045003 (2014). https://doi.org/10.1088/0264-9381/31/4/045003

35. G.V. Kraniotis, Class. Quant. Grav. 33(22), 225011 (2016). https:// doi.org/10.1088/0264-9381/33/22/225011

36. P.K. Townsend, Phys. Rev. D 15, 2795 (1977). https://doi.org/10. 1103/PhysRevD.15.2795

37. D. Amati, M. Ciafaloni, G. Veneziano, Phys. Lett. B 216, 41 (1989). https://doi.org/10.1016/0370-2693(89)91366-X
38. K. Konishi, G. Paffuti, P. Provero, Phys. Lett. B 234, 276 (1990). https://doi.org/10.1016/0370-2693(90)91927-4

39. L.J. Garay, Int. J. Mod. Phys. A 10, 145 (1995). https://doi.org/10. 1142/S0217751X95000085

40. G. Amelino-Camelia, Int. J. Mod. Phys. D 11, 35 (2002). https:// doi.org/10.1142/S0218271802001330

41. A. Kempf, G. Mangano, R.B. Mann, Phys. Rev. D 52, 1108 (1995). https://doi.org/10.1103/PhysRevD.52.1108

42. B. Chen, G. Li, X. Zu, J. Tang, J. Mod. Phys. 6(05), 578 (2015). https://doi.org/10.4236/jmp.2015.65063

43. P. Wang, H. Yang, S. Ying, Int. J. Theor. Phys. 55(5), 2633 (2016). https://doi.org/10.1007/s10773-015-2898-2

44. S. Capozziello, G. Lambiase, G. Scarpetta, Int. J. Theor. Phys. 39, 15 (2000). https://doi.org/10.1023/A:1003634814685

45. R.J. Adler, D.I. Santiago, Mod. Phys. Lett. A 14, 1371 (1999). https://doi.org/10.1142/S0217732399001462

46. F. Scardigli, G. Lambiase, E. Vagenas, Phys. Lett. B 767, 242 (2017). https://doi.org/10.1016/j.physletb.2017.01.054

47. S. Hossenfelder, M. Bleicher, S. Hofmann, J. Ruppert, S. Scherer, H. Stoecker, Phys. Lett. B 575, 85 (2003). https://doi.org/10.1016/ j.physletb.2003.09.040

48. K. Nozari, M. Karami, Mod. Phys. Lett. A 20, 3095 (2005). https:// doi.org/10.1142/S0217732305018517

49. T. Padmanabhan, Gravitation: Foundations and Frontiers (Cambridge University Press, Cambridge, 2010)

50. W. Greiner, Relativistic Quantum Mechanics: Wave equations (Springer, New York, 1990)

51. R.M. Wald, General Relativity (Chicago University Press, Chicago, 1984). https://doi.org/10.7208/chicago/9780226870373.001.0001

52. G. 't Hooft. Lecture notes on introduction to the theory of black holes (2009). http://www.staff.science.uu.nl/ hooft101/lectures/ blackholes/BH_lecturenotes.pdf. Accessed 14 Dec 2018

53. J.M. Bardeen, W.H. Press, S.A. Teukolsky, Astrophys. J. 178, 347 (1972). https://doi.org/10.1086/151796

54. C.W. Misner, K.S. Thorne, J.A. Wheeler, Gravitation (W. H. Freeman, San Francisco, 1973)

55. D. Chen, S. Yang, Int. J. Mod. Phys. A 22, 5173 (2007). https:// doi.org/10.1142/S0217751X07038207

56. R.B. Mann, Black Holes: Thermodynamics, Information, and Firewalls. SpringerBriefs in Physics ((Springer, New York, 2015). https://doi.org/10.1007/978-3-319-14496-2

57. R. Kerner, R.B. Mann, Phys. Lett. B 665(4), 277 (2008). https:// doi.org/10.1016/j.physletb.2008.06.012

58. I.A. Meitei, T.I. Singh, S.G. Devi, N.P. Devi, K.Y. Singh, Int. J. Mod. Phys. A 33(12), 1850070 (2018). https://doi.org/10.1142/ S0217751X18500707

59. Q.Q. Jiang, S.Q. Wu, X. Cai, Phys. Rev. D 73, 064003 (2006). https://doi.org/10.1103/PhysRevD.73.064003, https://doi.org/10. 1103/PhysRevD.73.069902 [Erratum: Phys. Rev. D 73, 069902 (2006)]

60. J.M. Bardeen, B. Carter, S.W. Hawking, Commun. Math. Phys. 31, 161 (1973). https://doi.org/10.1007/BF01645742

61. A.J.M. Medved, E.C. Vagenas, Mod. Phys. Lett. A 20, 1723 (2005). https://doi.org/10.1142/S0217732305018025

62. M. Arzano, A.J.M. Medved, E.C. Vagenas, JHEP 09, 037 (2005). https://doi.org/10.1088/1126-6708/2005/09/037

63. J. Zhang, Phys. Lett. B 668, 353 (2008). https://doi.org/10.1016/j. physletb.2008.09.005 